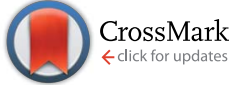

Cite this: RSC Adv., 2017, 7, 8460
Received 16th December 2016 Accepted 16th January 2017

DOI: $10.1039 / \mathrm{c} 6 \mathrm{ra} 28304 \mathrm{~g}$

www.rsc.org/advances

\section{Thermoelectric enhancement in sliver tantalate via strain-induced band modification and chemical bond softening}

\begin{abstract}
Jun $\mathrm{Li}^{\text {ab }}$ Zuju Ma ${ }^{\mathrm{b}}$ and Kechen $\mathrm{Wu}^{\text {*a }}$
The improvement of the thermoelectric performance (determined by the $Z T$ value) of materials is limited by the inter-correlation of the transport coefficients. By performing first principle calculations combined with semi-classical Boltzmann theory, we have systematically investigated the geometric, electronic and thermoelectric properties of $\mathrm{AgTaO}_{3}$ under external strain. It is demonstrated that the electronic structure and phonon transport can be modified independently. Compressive strain along the $c$-axis decreases the energy separation between the light and heavy valence bands and generates multiple valence band valleys near the Fermi level. Such band structure modification greatly enhances the Seebeck coefficient for the $p$ type doped compound, which is increased from $600 \mu \mathrm{V} \mathrm{K}^{-1}\left(\delta_{c}=0 \%\right)$ to $690 \mu \mathrm{V} \mathrm{K}^{-1}\left(\delta_{c}=-5 \%\right)$. Similarly, tensile strain softens the chemical bonding, favoring the reduction of thermal conductivity. Therefore, the improvement in the thermoelectric performance of $\mathrm{AgTaO}_{3}$ under strain suggests that strain engineering is a generally applicable route to enhancing the Seebeck coefficient and $Z T$ in thermoelectric materials.
\end{abstract}

\section{Introduction}

Thermoelectric materials, which can directly convert heat into electricity and vice versa, are of great importance in the search for clean and renewable energy sources..$^{1-4}$ The efficiency of thermoelectric materials is governed by the dimensionless thermoelectric figure of merit, $Z T=S^{2} \sigma T / \kappa$, where $S$ is the Seebeck coefficient, $\sigma$ is the electrical conductivity, $T$ is the absolute temperature, and $\kappa$ is the total thermal conductivity, consisting of lattice $\left(\kappa_{\mathrm{ph}}\right)$ and electronic $\left(\kappa_{\mathrm{el}}\right)$ contributions. There is no known upper bound on $Z T$, but the current energy crisis has driven the pursuit of higher performance materials with $Z T>1$ for commercial use. ${ }^{5,6}$ It is a challenging task to achieve high $Z T$ values, since all the electronic parameters $\left(\sigma, S\right.$ and $\left.\kappa_{\mathrm{el}}\right)$ are interrelated and their simultaneous optimization is difficult.

Current approaches to improve the $Z T$ value of thermoelectric materials can be classified into two categories: (i) enhancement of the electrical transport properties via modification of the band structure, such as distorting the lattice, ${ }^{7,8}$ doping with heteroatoms, ${ }^{9,10}$ or introducing quantum confinement effects; ${ }^{11-13}$ (ii) reduction of the thermal conductivity by introducing nanostructured components, ${ }^{14,15}$ layer structures, ${ }^{6,16}$ or alloying. ${ }^{17}$ The mechanism behind the band engineering strategy to increase $Z T$ involves the enhancement of the Seebeck coefficient by introducing a resonant state at the edge of the band, or increasing the

${ }^{a}$ State Key Laboratory of Structural Chemistry, Fujian Institute of Research on the Structure of Matter, Chinese Academy of Sciences, Fuzhou, Fujian 350002, People's Republic of China. E-mail: wkc@fjirsm.ac.cn

${ }^{b}$ University of Chinese Academy of Sciences, Beijing 100049, People's Republic of China convergence of electronic band. The low thermal conductivity originates from the strengthened phonon scattering of low dimensional or the strong anharmonic materials. Furthermore, the phonon interactions are interlinked with the bonding character, namely, a weak chemical bonding interaction gives rise to low thermal conductivity due to the lower speed of sound. ${ }^{18,19}$ Inspired by the two aspects of improving the thermoelectric performance, another effective method is strain-engineering. As previously reported, the application of pressure or strain has been shown to be a promising strategy to modify electronic structure and phonon transport, and thus tune the thermoelectric properties. For example, the Seebeck coefficient can be significantly enhanced by strain induced band structure distortion or band convergence. Moreover, the thermal conductivity of some materials can be reduced by applying strain..$^{20,21}$ Hence, it is interesting to study the effect of strain on electrical transport and thermal transport simultaneously, and thus the thermoelectric performance.

Oxide materials attract much attention as promising thermoelectric alternatives because of their advantages of nontoxicity and earth-abundance. For example, Zhang et al. reported that heavy doping ${ }^{22}$ and inducing $2 \mathrm{D}$ electron gas grain boundaries $^{23,24}$ could enhance the thermoelectric performance of $\mathrm{SrTiO}_{3}$. Furthermore, layered cobaltates ${ }^{25-27}$ and BiCuSeO systems $^{28,29}$ are expected to play important roles in thermoelectric applications due to their low thermal conductivity. Many oxide semiconductors with a primary structure of $\mathrm{ABO}_{3}$ have been shown to exhibit diverse physical properties such as superconductivity, ${ }^{30,31}$ optical transparency ${ }^{32,33}$ and photocatalytic activity, ${ }^{34,35}$ and have been reported to possess high 
mobility at room temperature. Tantalum oxide, $\mathrm{AgTaO}_{3}$, stands out amongst the multitudinous $\mathrm{ABO}_{3}$ type oxides due to its excellent carrier mobility. ${ }^{36}$ The $\mathrm{TaO}_{6}$ octahedral network contributes to high electrical mobility and a wide band gap, and has thus been attracting increasing attention in applications such as solar cells and even thermoelectric materials..$^{37-39}$

In this work, based on first-principles calculations combined with semi-classical Boltzmann transport theory, we systematically investigate the effect of uniaxial strain on the geometric, electronic and thermoelectric properties of $\mathrm{AgTaO}_{3}$. It is revealed that the compressive $c$-axial strain can greatly enhance the Seebeck coefficient of p-type doped $\mathrm{AgTaO}_{3}$, by modulating the valence band character. In addition, the tensile strain can increase the bond lengths of Ta and $\mathrm{O}$ atoms, as well as the unit cell volume, which is beneficial for softening the chemical bonds. Furthermore, the tensile uniaxial strain improves the electrical conductivity of p-type doped $\mathrm{AgTaO}_{3}$. The weakened chemical bonding interaction under tensile strain tends to reduce the bulk modulus, which contributes to a reduction in thermal conductivity.

\section{Computational details}

Calculations in this work were performed within density functional theory (DFT), using the projector augment-wave (PAW) method $^{40}$ incorporated in the Vienna Ab-initio Simulation Package (VASP). ${ }^{41,42}$ Generalized gradient approximation (GGA) with the Perdew-Burke-Ernzerhof (PBE) functional was used to describe the exchange-correlation interaction..$^{43} \mathrm{~A}$ kinetic energy cutoff of $500 \mathrm{eV}$ was selected for the plane wave expansion, and a Monkhorst-Pack $k$-point mesh of $6 \times 6 \times 4$ was used to sample the irreducible Brillouin zone (IBZ) in the structural optimization and self-consistent calculation. The total energy convergence was set to be less than $0.1 \mathrm{meV}$ between two steps. The lattice vectors and atomic positions were fully relaxed until the maximal Hellmann-Feynman forces acting on each atom were less than $0.001 \mathrm{eV} \mathrm{A}^{-1}$. It was found that the calculated electronic structure of $\mathrm{AgTaO}_{3}$ based on GGA/PBE, GGA/PW91 and LDA tends to be similar, and the band gap based on GGA $(1.92 \mathrm{eV})$ is closer to the experimental value than that based on LDA $(1.84 \mathrm{eV})$. Therefore, only the results obtained from GGA/PBE are shown. An exact band structure is of crucial importance for the accurate prediction of TE transport properties, because the Seebeck coefficient, electrical conductivity, and electronic contribution to the thermal conductivity depend on it. Therefore, a denser $k$-point grid of 21 $\times 21 \times 9$ was used for electronic structure calculation. The BoltzTrap program ${ }^{44}$ was employed to investigate the thermoelectric (TE) properties based on the analytical expressions of the electronic bands. The $k$-point mesh convergence test was performed to guarantee the accurate prediction of the TE properties.

\section{Results and discussion}

\section{Geometrical structure}

$\mathrm{AgTaO}_{3}$ crystallizes with a rhombohedral structure in the space group $R \overline{3} c$, as shown in Fig. 1a. The three-dimensional framework is composed of interlinked corner-sharing $\mathrm{TaO}_{6}$ octahedra, represented by shading. In every octahedral structure, the $\mathrm{Ta}-\mathrm{O}-\mathrm{Ta}$
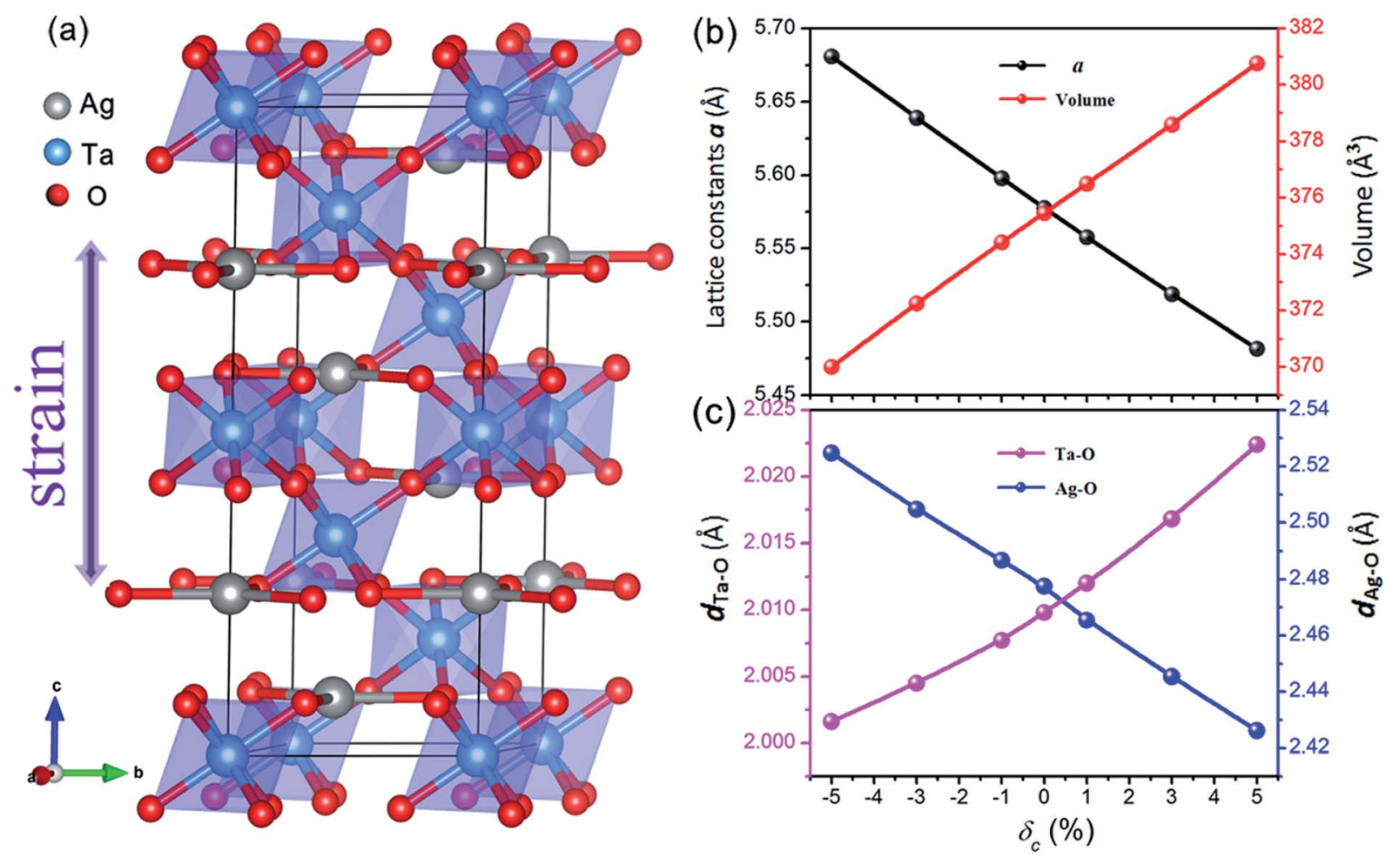

Fig. 1 (a) Conventional cell of strain-free $\mathrm{AgTaO}_{3}$. The polyhedron configuration of $\mathrm{TaO}_{6}$ is presented as shapes. Gray, blue and red spheres represent $\mathrm{Ag}$, Ta and $\mathrm{O}$ atoms, respectively. The strain is imposed along the $c$-axis direction. (b) The lattice constant $a$ and unit cell volume, and (c) bond length of $\mathrm{Ta}-\mathrm{O}$ and $\mathrm{Ag}-\mathrm{O}$ as functions of $\mathrm{c}$-axial strain. 
bonding angle is close to 180 degrees, which gives rise to the excellent carrier mobility of the $\mathrm{AgTaO}_{3}$ system. The lattice parameters of the relaxed cell of unstrained $\mathrm{AgTaO}_{3}$ are $a=5.58$ $\AA$ and $c=13.94 \AA$, which are in agreement with the experimental results of $a=5.53 \AA$ and $c=13.72 \AA .^{45}$ Based on the equilibrium structure, external uniaxial strain along the $c$-axis is imposed. The strength of the $c$-axial strain is defined as $\delta_{c}=\left(c-c_{0}\right) / c_{0} \times 100 \%$, where $c$ and $c_{0}$ are the lattice parameters along the $z$-direction with and without strain, respectively. Positive and negative $\delta$ corresponds to tensile and compressive strain, respectively, while $\delta_{c}=0$ means a strain-free case. The parameter $a$ and all the atomic positions are fully relaxed by minimization of the total energy. The calculated independent elastic constants for both with and without strained compounds are all satisfied the BornHuang stability criteria of a rhombohedral crystal. ${ }^{46}$

The effect of the $c$-axial strain on the lattice parameter $a$ and unit cell volume are presented in Fig. 1b. The lattice parameter $a$ decreases gradually with increasing $c$-axial strain from $-5 \%$ to $5 \%$, leading to a normal positive Poisson ratio, which is similar to most strained materials. As expected, the negative strain compresses the lattice volume, while the tensile strain expands it. The bond lengths of $\mathrm{Ta}-\mathrm{O}$ and $\mathrm{Ag}-\mathrm{O}$ exhibit a completely inverse dependence on the uniaxial strain, as shown in Fig. 1c. The Ta-O bond expands from $2.002 \AA$ to $2.022 \AA$ with an increase in strain from $-5 \%$ to $5 \%$, while the $\mathrm{Ag}-\mathrm{O}$ bond shrinks from $2.525 \AA$ to $2.426 \AA$. Nevertheless, all of the $\mathrm{Ag}-\mathrm{O}$ bonds are parallel to the $a b$ plane and the Ta-O bonds organize the framework of $\mathrm{AgTaO}_{3}$, indicating that the Ta-O bond dominates the global bonding interaction. The expanded unit cell volume and Ta-O bond length under tensile strain account for the chemical bond weakening, which contributes to a reduction in the thermal conductivity.

\section{Electronic structure}

The transport properties of thermoelectric materials closely depend on the electronic states. The curvatures of the conduction and valence bands near the Fermi level determine the carrier transport, and thus affect the thermoelectric power and electrical

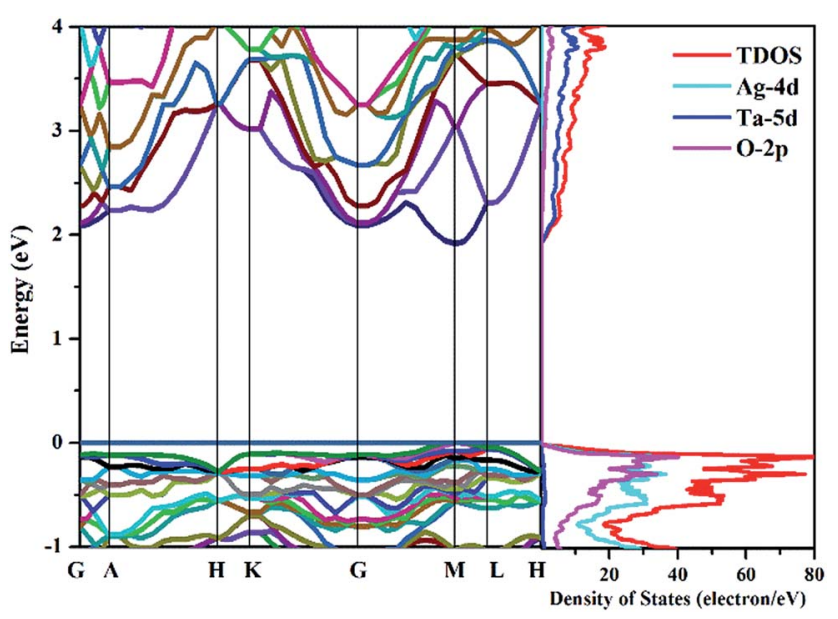

Fig. 2 Band structures of optimized $\mathrm{AgTaO}_{3}$ (left) and the total and partial density of states (right). The energy zero is at the valence-band maximum. conductivity. In order to understand the electronic characteristics of $\mathrm{AgTaO}_{3}$, the calculated band structure and density of states (DOS) are shown in Fig. 2. The calculated band gap of unstrained $\mathrm{AgTaO}_{3}$ is $1.92 \mathrm{eV}$, which is an underestimation compared to the experimental value of $3.4 \mathrm{eV},{ }^{47}$ due to the well-known limitations of the GGA exchange functional. $\mathrm{AgTaO}_{3}$ is a direct band gap semiconductor with both the valence band maximum (VBM) and the conduction band minimum (CBM) located at the $M$ point. It can be observed that the valence bands and conduction bands possess significantly different characteristics, indicating the different thermoelectric performances of $\mathrm{p}$ - and n-type doped $\mathrm{AgTaO}_{3}$. The flat bands near the VBM contribute to a heavy effective mass, giving rise to high thermoelectric power but small conductivity for $\mathrm{p}$-type doping. In contrast, the high dispersive conduction band indicates a small effective mass near the CBM, which favors high electrical conductivity in n-type compounds. This phenomenon can be further demonstrated with the corresponding DOS. It can be found that the valence band consists predominately of a mixture of $\mathrm{Ag}-4 \mathrm{~d}$ and $\mathrm{O}-2 \mathrm{p}$ states, while the conduction band is dominated mainly by Ta- $5 \mathrm{~d}$ states. Typically, thermoelectric materials with large Seebeck coefficients are usually associated with a large DOS near the band gap. Therefore, the higher DOS peak nearer the VBM than the CBM of $\mathrm{AgTaO}_{3}$ is indicative of a higher Seebeck coefficient for p-type doping than that for n-type doping.

Now let us address the effect of the $c$-axial strain on the electronic structure. The band structure of $\mathrm{AgTaO}_{3}$ under compressive strain $\left(\delta_{c}=-5 \%\right.$ and $\left.\delta_{c}=-3 \%\right)$ and tensile strain $\left(\delta_{c}=5 \%\right.$ and $\delta_{c}$ $=3 \%)$ are plotted in Fig. 3, and that of the unstrained one $\left(\delta_{c}=0\right)$ is also presented for comparison. For clarity, we respectively extracted and enlarged the details of the conduction band (Fig. $3 \mathrm{a}_{1}-\mathrm{e}_{1}$ ) and valence band (Fig. $3 \mathrm{a}_{2}-\mathrm{e}_{2}$ ) under different strains. In general, the response of the band structure to the compressive strain is stronger than that to the tensile strain, especially for the valence band. When compressive strain is imposed, the conduction band edge at the $\Gamma$ point splits gradually, resulting in the CBM transfering from the $M$ point to the $\Gamma$ point (Fig. $3 \mathrm{a}_{1}$ and $\mathrm{b}_{1}$ ). Consequently, the energy band gap $\left(E_{\mathrm{g}}\right)$ remains almost unchanged under a strain of $-3 \%$ but is greatly decreased at $-5 \%$, due to the transformation of the CBM. Therefore, the compressive strain can cause $\mathrm{AgTaO}_{3}$ to transform from a direct to an indirect semiconductor. The tensile strain does not turn the energy gap from direct to indirect, but decreases the $E_{\mathrm{g}}$ from $1.92 \mathrm{eV}$ to $1.75 \mathrm{eV}$. However, when the tensile strain reaches $5 \%$, the conduction band at the $\Gamma$ point splits significantly, forming two similar band valleys at the $\Gamma$ and $M$ points (Fig. $3 \mathrm{e}_{1}$ ). For the valence band (Fig. $3 \mathrm{a}_{2}-\mathrm{e}_{2}$ ), the compressive strain modifies the valence band by reducing the energy offset between the light and heavy hole valence bands, forming a multiple-valley valence band near the Fermi energy level. Thus the compressive strain results in an improvement of the valence band degeneracy, which favors the enhancement of the Seebeck coefficient for p-type doping, as has been demonstrated for hole-doped SnSe by Zhao et $a .^{48}$ The tensile strain enhances the band dispersion at the VBM by reducing the band energy of other symmetry points, which contributes to the enhancement of the electrical conductivity for p-type doping. 

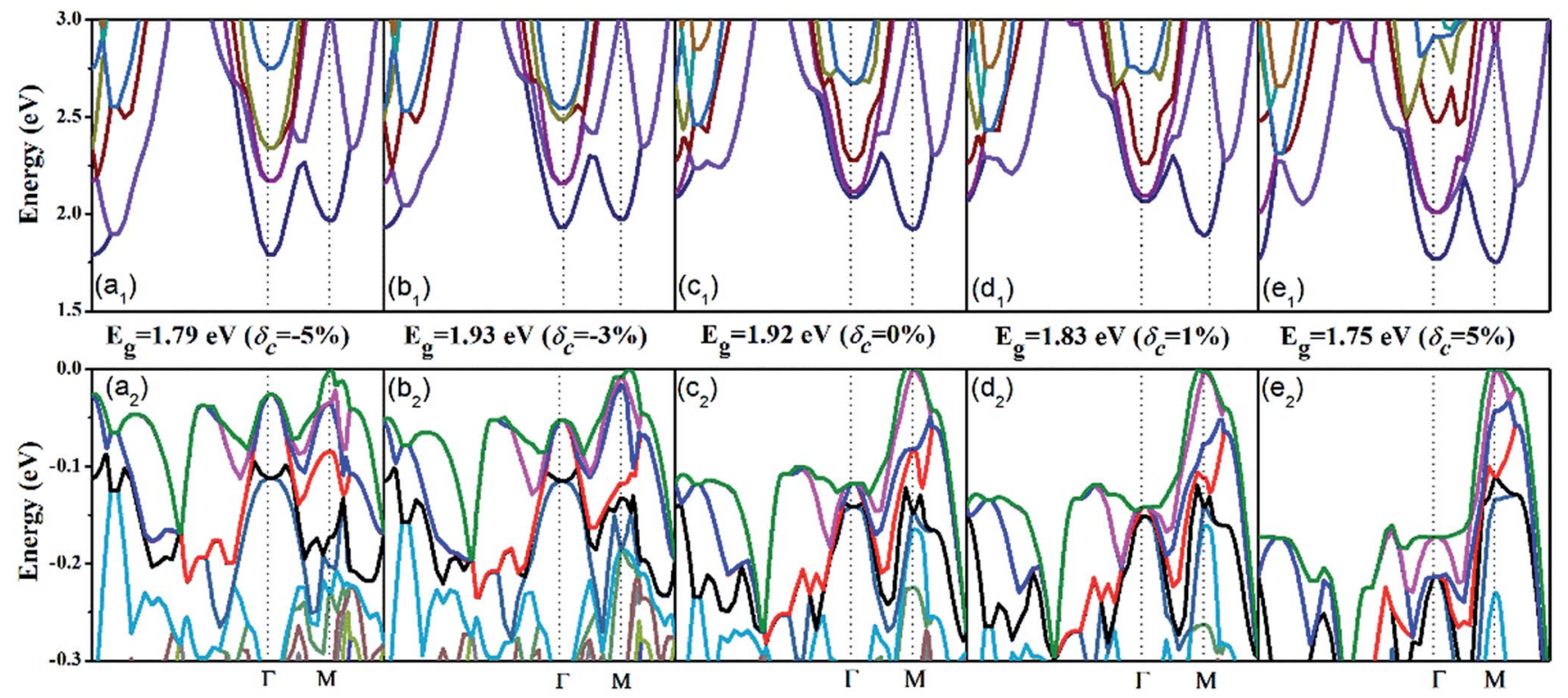

Fig. 3 The electronic band structure under different stains: $\left(a_{1}-e_{1}\right)$ the conduction band and $\left(a_{2}-e_{2}\right)$ the valence band.

To illustrate the effect of strain on the electronic structure near the Fermi level in more detail, we collected the total DOS of the valence band (from $-0.25 \mathrm{eV}$ to $0 \mathrm{eV}$ ) and the conduction band (from $1.70 \mathrm{eV}$ to $2.20 \mathrm{eV}$ ) under strain as shown in Fig. 4, with the zero-energy point representing the respective VBMs. The asymmetric increase in the valence band density of states near the Fermi level is in accordance with the valence band degeneracy, giving rise to an increased Seebeck coefficient and power factor for p-type doping. However, although the tensile strain increases the density of states of the CBM, the magnitude of the value is not on par with the DOS value of the VBM. Hence, the Seebeck coefficient for n-type doping is weakly dependent on strain, except for when $\delta_{c}=-5 \%$. Under the compressive strain of $\delta_{c}=-5 \%$, the abnormal conduction DOS character induces an obvious decrease in the Seebeck coefficient, as depicted below.

\section{Thermoelectric properties}

Based on the parameters from the calculated electronic structures, the thermoelectric properties of $\mathrm{AgTaO}_{3}$ can be calculated by solving the Boltzmann transport equation using the BolazTrap code. To examine the effect of uniaxial strain on the ability to produce a voltage from the temperature difference, the Seebeck coefficients of the $\mathrm{p}$ - and n-type doped compounds under different strains, as a function of hole and electron concentration at $T=300 \mathrm{~K}$, are presented in Fig. 5 a and b. It can be found that

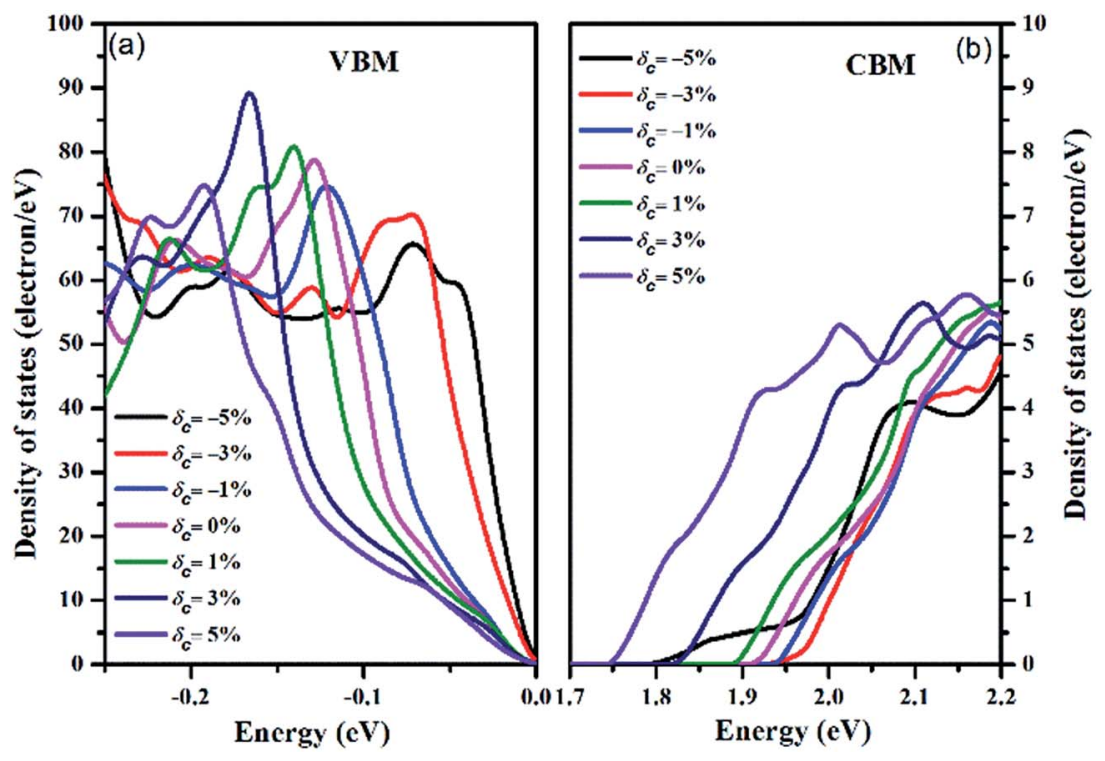

Fig. 4 The density of states under different strains for the (a) VBM and (b) CBM. The Fermi energy level is shifted to $0 \mathrm{eV}$. 

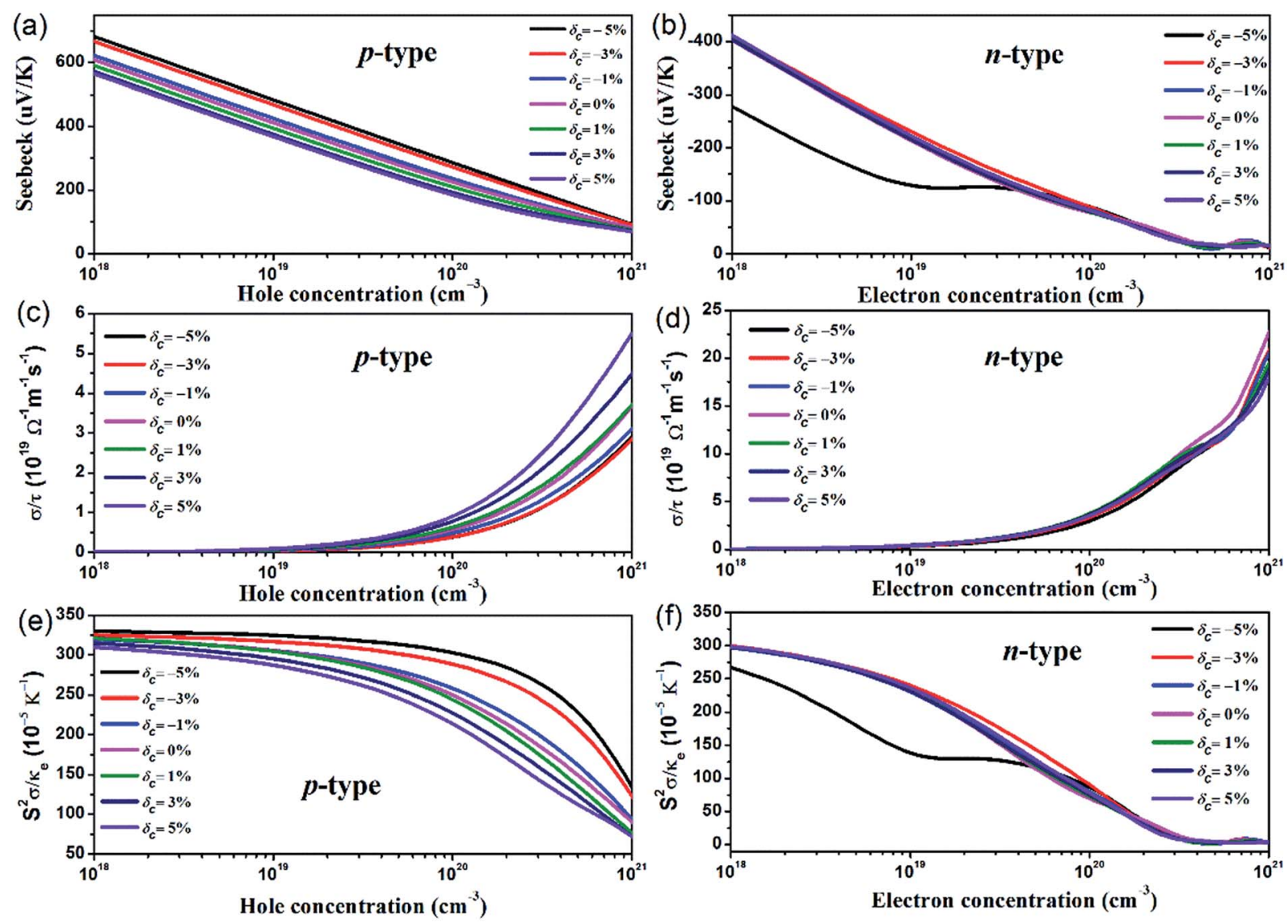

Fig. 5 Calculated electronic transport parameters: $S(\mathrm{a})$ and (b), $\sigma$ (c) and (d), $s^{2} \sigma / \kappa_{\mathrm{e}}$ (e) and (f) as a function of hole concentration (p-type) and electron concentration (n-type) under different uniaxial strains along the $c$-axis from $-5 \%$ to $5 \%$.

for both the p- and n-type doped compounds, the Seebeck coefficient exhibits a near linear decreasing trend with increasing carrier concentration. For p-type doping (Fig. 5a), the compressive strain brings an obvious enhancement to the Seebeck coefficient. With the hole concentration at $10^{18} \mathrm{~cm}^{-3}$, the Seebeck coefficient increases from $600 \mu \mathrm{V} \mathrm{K}{ }^{-1}\left(\delta_{c}=0 \%\right)$ to $690 \mu \mathrm{V} \mathrm{K}^{-1}\left(\delta_{c}\right.$ $=-5 \%)$. The remarkable enhancement originates from the multiple-valley valence band and the pronounced band asymmetry near the Fermi level induced by the compressive strain. For n-type doping, the Seebeck coefficient exhibits a weak dependence on the $c$-axial strain, except for the case of $\delta_{c}=-5 \%$. At a small doping level with an electron concentration of lower than $\sim 4 \times 10^{19} \mathrm{~cm}^{-3}$, a compressive strain of $\delta_{c}=-5 \%$ decreases the Seebeck coefficient significantly, which can be ascribed to the abnormal characteristics of the conduction band under these conditions. Comparing the two kinds of doping style, at the same hole doping and electron doping concentration, the Seebeck coefficient of p-type doped $\mathrm{AgTaO}_{3}$ is much higher than that of the n-type doped $\mathrm{AgTaO}_{3}$ due to the high valence band DOS peak, which manifests as improved thermoelectric properties of $\mathrm{AgTaO}_{3}$ as a p-type material.

The directly obtained electrical conductivities from the BoltzTrap code are expressed as the ratio of the electrical conductivity $(\sigma)$ to the scatting time $\tau, \sigma / \tau$. The constant scatting time approximation is always adopted when $\sigma$ is calculated, thus the effect of strain on electrical conductivity can be demonstrated by the effect of strain on the value of $\sigma / \tau$. As presented in Fig. $5 \mathrm{c}$ and d, the $\sigma / \tau$ value for both p- and n-type doped $\mathrm{AgTaO}_{3}$ increases with the hole and electron concentration increasing from $10^{18} \mathrm{~cm}^{-3}$ to $10^{21} \mathrm{~cm}^{-3}$. However, the small electron effective mass due to the light and high dispersive conduction band contributes to a large electrical conductivity value for n-type doped $\mathrm{AgTaO}_{3}$. For p-type doping, the value of $\sigma / \tau$ can be obviously enhanced by applying tensile strain but is decreased slightly under compressive strain. This can be easily explained using the electronic band structure under different strains: the valence band dispersion near the Fermi energy level is strengthened by the tensile strain, which produces a small hole effective mass, resulting in the enhancement of electrical conductivity for p-type doping. For ntype doping, similar to the Seebeck coefficient, the $\sigma / \tau$ is weakly sensitive to the uniaxial strain, as shown in Fig. $5 \mathrm{~d}$.

The Seebeck coefficient, electrical conductivity and electrical thermal conductivity are interlinked and determined by the electronic transport processes, thus the value of $S^{2} \sigma / \kappa_{\mathrm{e}}$ is much better for discussing the thermoelectric properties, due to the absence of scattering time. Consequently, the effect of strain on the value of $S^{2} \sigma / \kappa_{\mathrm{e}}$ for p-type and n-type doping as a function of strain is plotted in Fig. $5 \mathrm{e}$ and $\mathrm{f}$. As described above, the effects of the carrier concentration on the Seebeck coefficient and the 


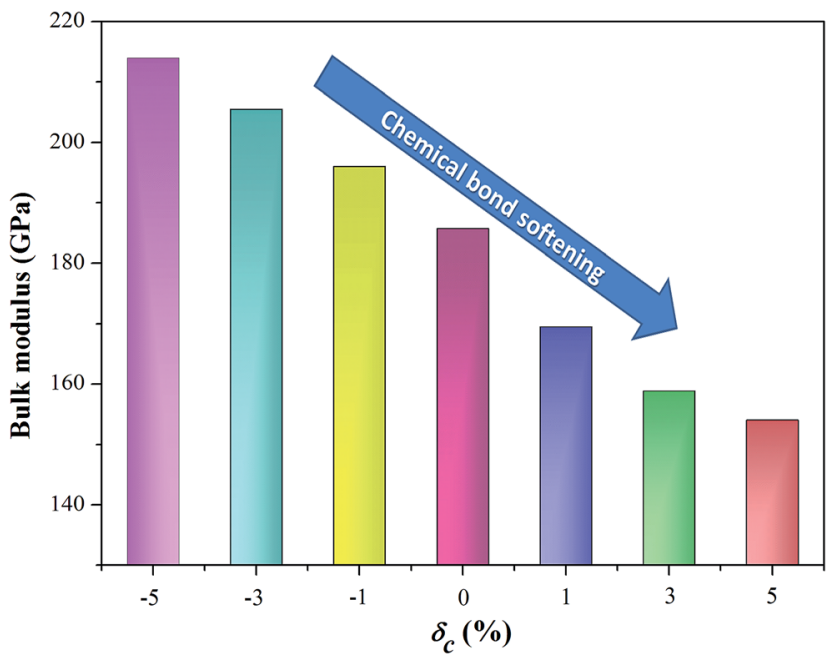

Fig. 6 A schematic illustration of the bulk modulus of $\mathrm{AgTaO}_{3}$ under different $c$-axial strains.

electrical conductivity are opposite. Accordingly, a compromise between $S$ and $\sigma$ should be taken into consideration, in order to obtain an optimized thermoelectric performance. For p-type doping, the $S^{2} \sigma / \kappa_{\mathrm{e}}$ value monotonously increases with an increase in compressive strain, and this is more obvious at the heavy hole doping level. Contrarily, the tensile strain decreases the $S^{2} \sigma / \kappa_{\mathrm{e}}$ value gradually, induced by the degenerated Seebeck coefficient. For n-type doping, the $S^{2} \sigma / \kappa_{\mathrm{e}}$ value is nearly unaffected by the strain, except for the case of $\delta_{c}=-5 \%$, due to the dramatic response of the Seebeck coefficient to the compressive strain of $-5 \%$.

The transport parameters we discussed above describe all electronic function. Another important parameter that determines the thermoelectric performance is the lattice thermal conductivity, which depends on phonon transport. Phonon interactions are interlinked with the bonding character and strength. As mentioned when discussing the effect of strain on geometric structure, the unit cell volume expands under the $c$ axial tensile strain (as shown in Fig. 1b), which weakens the global chemical bonding interaction of $\mathrm{AgTaO}_{3}$. This can also be confirmed from the simulated bulk modulus of a compound under uniaxial strain, as visualized in Fig. 6 . The bulk modulus decreases with an increase in the $c$-axial strain from $-5 \%$ to $5 \%$, which is considered to be due to the gradual softening of the bonds under the rising tensile strain. The bond softening, i.e. the chemical bond interaction weakening, usually means a low phonon velocity which causes strong phonon scatterings, leading to reduced thermal conductivity.

\section{Conclusions}

In summary, we systematically investigated the effect of strain on the geometric, electronic, and p-type/n-type doped thermoelectric properties of $\mathrm{AgTaO}_{3}$. The results show that strain engineering can modify the electronic transport and phonon transport independently, which is expected to be an effective way to improve the thermoelectric performance. The compressive strain leads to the degeneracy of the valence band and increases the density of states of the VBM, giving rise to the enhancement of the Seebeck coefficient and power factor for $\mathrm{p}$ type doping. The tensile strain enlarges the volume of the unit cell and decreases the bulk modulus of $\mathrm{AgTaO}_{3}$, which induces bond softening. The weakened chemical bonding interaction is favorable to phonon softening, which is conducive to the reduction of thermal conductivity. The present study offers hints that the thermoelectric performance of $\mathrm{AgTaO}_{3}$ can be enhanced by applying external strain.

\section{Acknowledgements}

This work was supported by the National Science Foundation of China (No. 21501177). We acknowledge the Supercomputing Center of CNIC and Special Program for Applied Research on Super Computation of the National Science Foundation of China-Guangdong Joint Fund (the second phase) for providing the computer resources.

\section{References}

1 R. Venkatasubramanian, E. Siivola, T. Colpitts and B. O'Quinn, Nature, 2001, 413, 597-602.

2 G. J. Snyder and E. S. Toberer, Nat. Mater., 2008, 7, 105-114. 3 S.-J. Kim, J.-H. We and B.-J. Cho, Energy Environ. Sci., 2014, 7, 1959-1965.

4 G. Tan, L.-D. Zhao and M. G. Kanatzidis, Chem. Rev., 2016, 116, 12123-12149.

5 H.-J. Wu, L.-D. Zhao, F.-S. Zheng, D. Wu, Y.-L. Pei, X. Tong, M. G. Kanatzidis and J.-Q. He, Nat. Commun., 2014, 5, 4515. 6 L.-D. Zhao, S.-H. Lo, Y.-S. Zhang, H. Sun, G.-J. Tan, C. Uher, C. Wolverton, V. P. Dravid and M. G. Kanatzidis, Nature, 2014, 508, 373-377.

7 J. P. Heremans, V. Jovovic, E. S. Toberer, A. Saramat, K. Kurosaki, A. Charoenphakdee, S. Yamanaka and G. J. Snyder, Science, 2008, 321, 554-557.

8 W. Liu, X.-J. Tan, K. Yin, H.-J. Liu, X.-F. Tang, J. Shi, Q.-J. Zhang and C. Uher, Phys. Rev. Lett., 2012, 108, 166601.

9 Y.-B. Luo, J.-Y. Yang, M. Liu, Y. Xiao, L.-W. Fu, W.-X. Li, D. Zhang, M.-Y. Zhang and Y.-D. Cheng, J. Mater. Chem. A, 2015, 3, 1251-1257.

10 J.-S. Rhyee, K.-H. Ahn, K.-H. Lee, H.-S. Ji and J.-H. Shim, Adv. Mater., 2011, 23, 2191-2194.

11 L. Hicks and M. Dresselhaus, Phys. Rev. B: Condens. Matter Mater. Phys., 1993, 47, 16631-16634.

12 L. D. Hicks and M. S. Dresselhaus, Phys. Rev. B: Condens. Matter Mater. Phys., 1993, 47, 12727-12731.

13 Y. Tian, M. R. Sakr, J. M. Kinder, D. Liang, M. J. MacDonald, R. L. J. Qiu, H.-J. Gao and X. P. A. Gao, Nano Lett., 2012, 12, 6492-6497.

14 W. Kim, J. Zide, A. Gossard, D. Klenov, S. Stemmer, A. Shakouri and A. Majumdar, Phys. Rev. Lett., 2006, 96, 045901.

15 X. Huang, C. Zhi, P. Jiang, D. Golberg, Y. Bando and T. Tanaka, Adv. Funct. Mater., 2013, 23, 1824-1831. 
16 C. Chiritescu, D. G. Cahill, N. Nguyen, D. Johnson, A. Bodapati, P. Keblinski and P. Zschack, Science, 2007, 315, 351-353.

17 D. M. Rowe, V. S. Shukla and N. Savvides, Nature, 1981, 290, 765-766.

18 S. Wang, Y. Sun, J. Yang, B. Duan, L. Wu, W. Zhang and J. Yang, Energy Environ. Sci., 2016, 9, 3436-3447.

19 P. Ying, X. Li, Y. Wang, J. Yang, C. Fu, W. Zhang, X. Zhao and T. Zhu, Adv. Funct. Mater., 2016, 27, 1604145.

20 Q.-X. Pei, Y.-W. Zhang, Z.-D. Sha and V. B. Shenoy, J. Appl. Phys., 2013, 114, 033526.

21 L.-Y. Zhu, T.-T. Zhang, Z.-M. Sun, J.-H. Li, G.-B. Chen and S. A. Yang, Nanotechnology, 2015, 26, 465707.

22 R.-Z. Zhang, C.-L. Wang, J.-C. Li, W.-B. Su, J.-L. Zhang, M.-L. Zhao, J. Liu, Y.-F. Zhang and L.-M. Mei, Solid State Sci., 2010, 12, 1168-1172.

23 R.-Z. Zhang, C.-L. Wang, J.-C. Li and K. Koumoto, J. Am. Ceram. Soc., 2010, 93, 1677-1681.

24 R.-Z. Zhang and K. Koumoto, J. Electron. Mater., 2013, 42, 1568-1572.

25 A. Maignan, S. Hebert, D. Pelloquin, C. Michel and J. Hejtmanek, J. Appl. Phys., 2002, 92, 1964-1967.

26 D. O. Demchenko and D. B. Ameen, Comput. Mater. Sci., 2014, 82, 219-225.

27 K. Takahata, Y. Iguchi, D. Tanaka, T. Itoh and I. Terasaki, Phys. Rev. B: Condens. Matter Mater. Phys., 2000, 61, 12551.

28 L.-D. Zhao, J. He, D. Berardan, Y. Lin, J.-F. Li, C.-W. Nan and N. Dragoe, Energy Environ. Sci., 2014, 7, 2900-2924.

29 J.-H. Sui, J. Li, J.-Q. He, Y.-L. Pei, D. Berardan, H.-J. Wu, N. Dragoe, W. Cai and L.-D. Zhao, Energy Environ. Sci., 2013, 6, 2916-2920.

30 M. H. K. Rubel, A. Miura, T. Takei, N. Kumada, M. M. Ali, M. Nagao, S. Watauchi, I. Tanaka, K. Oka, M. Azuma, E. Magome, C. Moriyoshi, Y. Kuroiwa and A. K. M. A. Islam, Angew. Chem., Int. Ed., 2014, 53, 3599-3603. 31 M. H. K. Rubel, T. Takei, N. Kumada, M. M. Ali, A. Miura, K. Tadanaga, K. Oka, M. Azuma, M. Yashima, K. Fujii,
E. Magome, C. Moriyoshi, Y. Kuroiwa, J. R. Hester and M. Aydeev, Chem. Mater., 2016, 28, 459-465.

32 S. De Wolf, J. Holovsky, S.-J. Moon, P. Loper, B. Niesen, M. Ledinsky, F.-J. Haug, J.-H. Yum and C. Ballif, J. Phys. Chem. Lett., 2014, 5, 1035-1039.

33 J. Zhu, Z. Xia, Y. Zhang, M. S. Molokeev and Q. Liu, Dalton Trans., 2015, 44, 18536-18543.

34 R. Asai, H. Nemoto, Q. Jia, K. Saito, A. Iwase and A. Kudo, Chem. Commun., 2014, 50, 2543-2546.

35 C. Clavero, Nat. Photonics, 2014, 8, 95-103.

36 M. Wiegel, M. H. J. Emond, E. R. Stobbe and G. Blasse, J. Phys. Chem. Solids, 1994, 55, 773-778.

37 H. Kato, H. Kobayashi and A. Kudo, J. Phys. Chem. B, 2002, 106, 12441-12447.

38 J.-T. Li and N.-Q. Wu, Catal. Sci. Technol., 2015, 5, 1360-1384. 39 A. Mahmood, S. M. Ramay, H. M. Rafique, Y. Al-Zaghayer and S. U. D. Khan, Mod. Phys. Lett. B, 2014, 28, 1450077.

40 P. E. Blöchl, Phys. Rev. B: Condens. Matter Mater. Phys., 1994, 50, 17953-17979.

41 G. Kresse and J. Furthmüller, Phys. Rev. B: Condens. Matter Mater. Phys., 1996, 54, 11169-11186.

42 G. Kresse and D. Joubert, Phys. Rev. B: Condens. Matter Mater. Phys., 1999, 59, 1758.

43 J. P. Perdew, K. Burke and M. Ernzerhof, Phys. Rev. Lett., 1996, 77, 3865-3868.

44 G. K. H. Madsen and D. J. Singh, Comput. Phys. Commun., 2006, 175, 67-71.

45 M. Wolcyrz and M. Lukaszewski, Z. Kristallogr., 1986, 177, 53-58.

46 M. Born and K. Huang, Dynamical theory of crystal lattices, Oxford university press, 1998.

47 H. Kato, H. Kobayashi and A. Kudo, J. Phys. Chem. B, 2002, 106, 12441-12447.

48 L.-D. Zhao, G.-J. Tan, S.-Q. Hao, J.-Q. He, Y.-L. Pei, H. Chi, H. Wang, S.-K. Gong, H.-B. Xu, V. P. Dravid, C. Uher, G. J. Snyder, C. Wolverton and M. G. Kanatzidis, Science, 2016, 351, 141-144. 